\title{
Primary Hepatic Leiomyosarcoma A Space Occupying Lesion in the Liver: An Enigma for Diagnosis
}

\author{
Rajdeep B More, Irfan A Shera*, Sitendu K Patel, Ashwini Kumar Setya and Vivek Raj \\ Department of Gastroenterology, Max Super Speciality Hospital, 1,2 Press Enclave Road, Saket, New Delhi - 110017, \\ India
}

Received: June 06, 2017; Accepted: July 31, 2017; Published: August 17, 2017

*Corresponding author: Irfan A Shera, Department of Gastroenterology, Max Super Speciality Hospital, 1,2 Press Enclave Road, Saket, New Delhi 110017, India, Tel: +918588818091; E-mail: sherairfan@gmail.com

\begin{abstract}
Primary hepatic leiomyosarcoma are rare soft tissue sarcomas with about only about 60 cases reported in the English literature [1]. Due to its rare existence and less studied, the diagnostic algorithms and standards of care have not been adequately defined. This has led to a delayed diagnosis and subsequently a poor prognosis and survival of such patients. We herein present a 72 year old lady with primary hepatic leiomyosarcoma treated with chemotherapy and attempt to delineate the line of management after reviewing the medical literature. Primary Hepatic Leiomyosarcoma is a rare primary hepatic malignancy which is usually a diagnosis of exclusion. The standard treatment guidelines are yet to be established; large cohort database is needed for better understanding of disease behaviour and management.
\end{abstract}

Key words: Primary Hepatic Leiomyosarcoma; Vimentin; Smooth muscle actin;

Abbreviations: PHL: Primary Hepatic Leiomyosarcomas; SMA: Smooth Muscle Actin; CT: Computed Tomography

\section{Introduction}

Primary hepatic leiomyosarcomas (PHL) account for 1-2\% of all liver malignancies. Due to the rarity of primary hepatic sarcomas, and PHL in particular, the diagnostic algorithms and standards of care have not been adequately defined. In worldwide literature there are only a few cases series are available. The standard management consensus guidelines for these tumors have not been yet established and most of the management strategies are based on expert opinion. This has led to a delayed diagnosis and subsequently a poor prognosis and survival of such patients. We herein present a 72 year old lady with primary hepatic leiomyosarcoma treated with chemotherapy and attempt to delineate the line of management after reviewing the English literature.

\section{Case Report}

A 72 year old Nigerian lady presented to our department with complaints of abdominal pain and swelling in the epigastrium and right hypochondrium over 3 months prior to presentation with other non specific symptoms such as generalized weakness, loss of appetite and weight loss. Her past medical and surgical history was insignificant. Physical examination revealed a massive hepatomegaly extending $6 \mathrm{~cm}$ beyond the costal margin in the midclavicular line. The swelling was bosselated and margin of the liver was irregular. There was no evidence of free fluid in the abdomen and the rest of the general and systemic examination was essentially normal.

Her baseline laboratory investigations like complete blood count, liver function test and coagulation profile were normal. Her serology for Hepatitis B, C and HIV I \& II was negative and tumour markers such as Alfa feto protein, CA 19.9, CEA, Ca-125 were within the normal range. Furthermore, the patient was subjected to a triple phase contrast computed tomography (CT) scan of the abdomen to evaluate for space occupying lesion of the liver. It showed irregular large heterogeneous lesion in segment 4 and 3 of left lobe of liver causing bulging of the anterior liver margin and showing heterogeous early arterial enhancement with interspersed non enhancing areas (cystic/necrotic). These lesions do not show washout and become isointense to the liver parenchyma in the portal venous phase as shown in (Figure $1 \mathrm{a}, \mathrm{b}$ ). CT demonstrated a massive hepatomegaly (Figure 2) enlarged left lobe of liver reaching up to the pelvis with a large heterogeneous lesion within it displacing the bowel loops inferiorly. Further imaging inform of PET-CT was done to evaluate metabolic activity and distance spread (Figure 3). In view of a normal Alfa feto protein and absence of washout in portal phase of triple phase CT abdomen with contrast, the patient was subjected to a true cut biopsy of the FDG avid liver lesion.
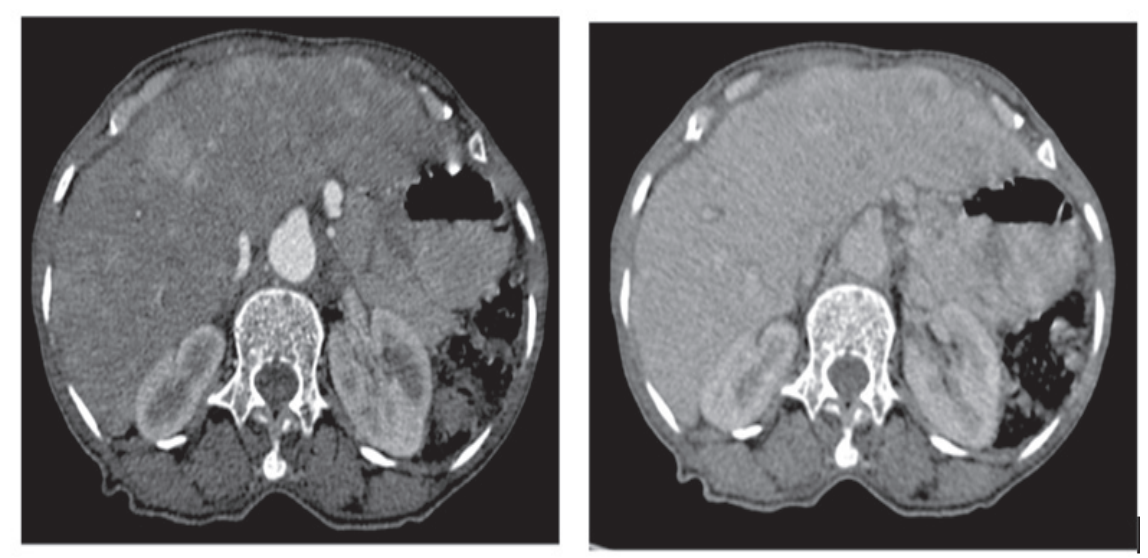

Figure $1 \mathbf{a}$ a b: Liver is studded with multiple nodules of varying sizes showing early arterial enhancement which become isointense to the liver parenchyma in P-V phase

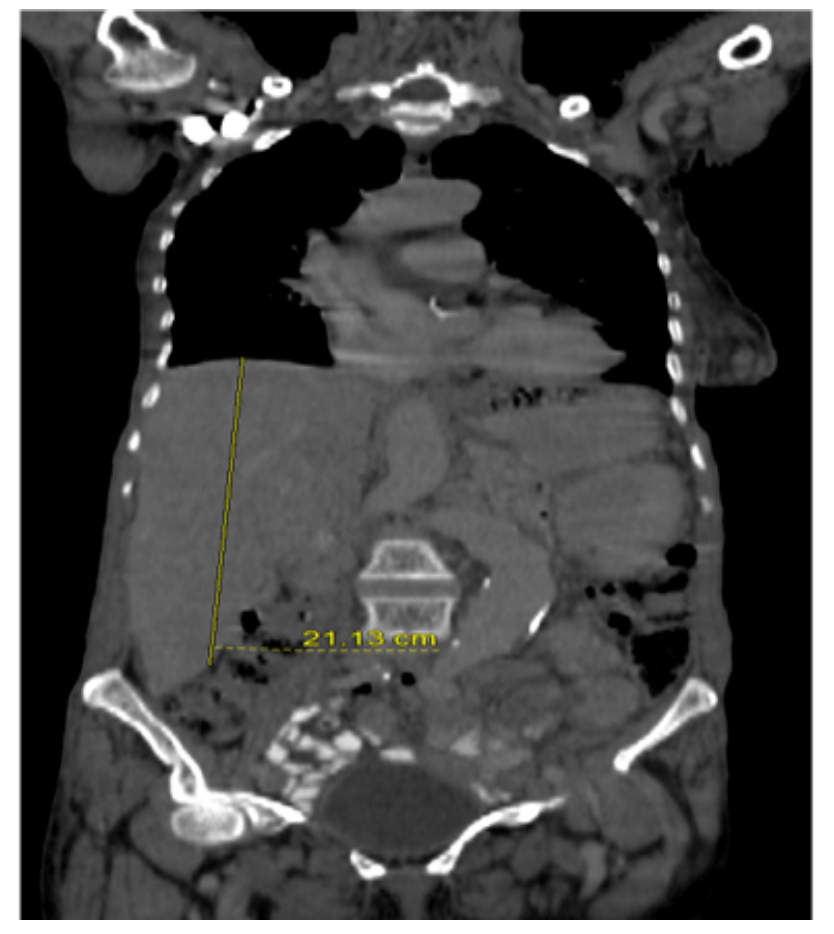

Figure 2: Coronal reformatted images showing gross hepatomegaly with irregular hepatic margins 


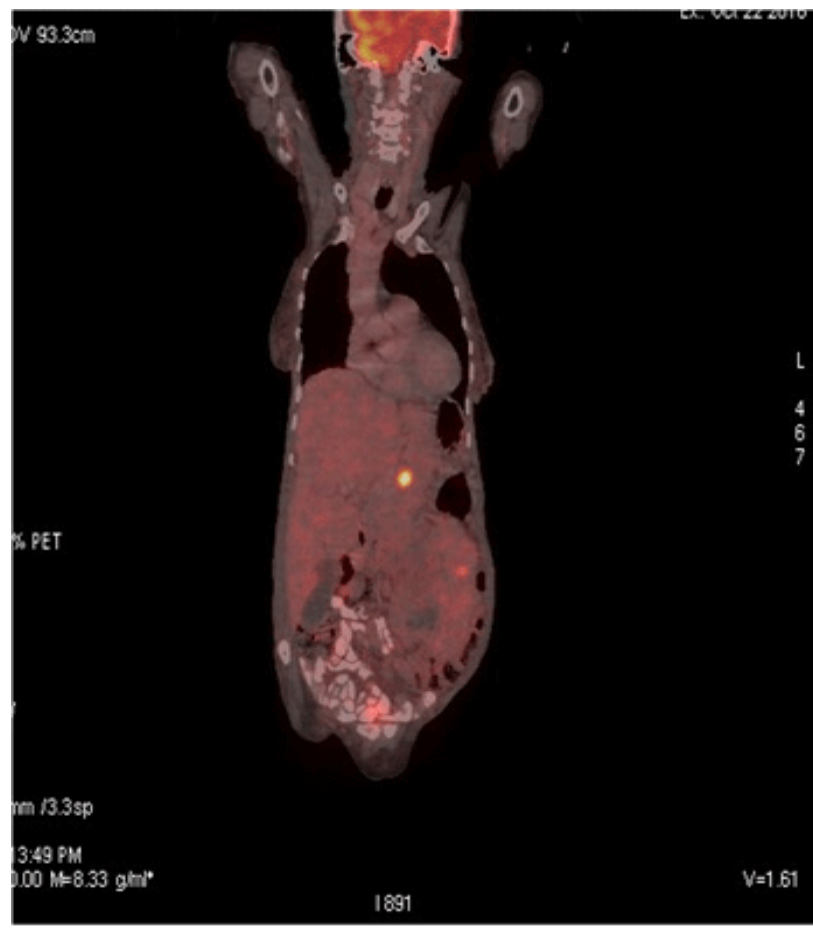

Figure 3: CPET - CT image showing FDG avid lesion in left lobe of liver

The Liver biopsy revealed that hepatic parenchymal infiltration by a highly cellular spindle cell tumor with moderate nuclear atypia and fair number of mitosis. The tumour cells had vesicular nuclei and prominent nucleoli with no evidence of necrosis (Figure 4). The diagnosis of spindle cell sarcoma of liver was made. Further characterization of tumour pathology immunohistochemistry was conducted. Vimentin and SMA (smooth muscle actin): positive, CK, Desmin S-100, CD34, CD117: negative and CD31: negative, H-Caldesmon-patchy positive Ki67 proliferation index: 40\%, Alk-1:negative.Final diagnosis of primary hepatic leiomyosarcoma was established (Figure 5).

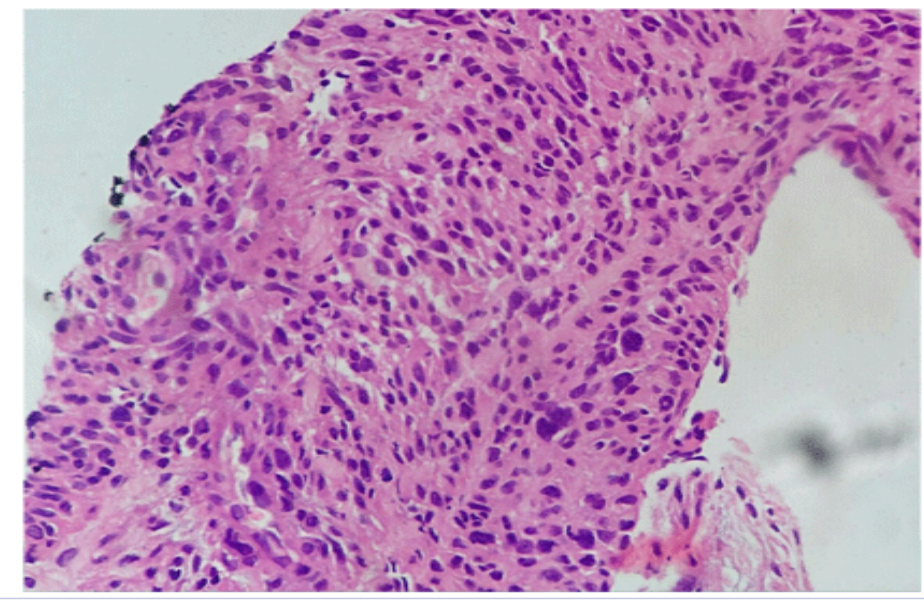

Figure 4: Hepatic parenchymal infiltration by a highly cellular spindle cell tumor with moderate nuclear atypia and fair number of mitosis

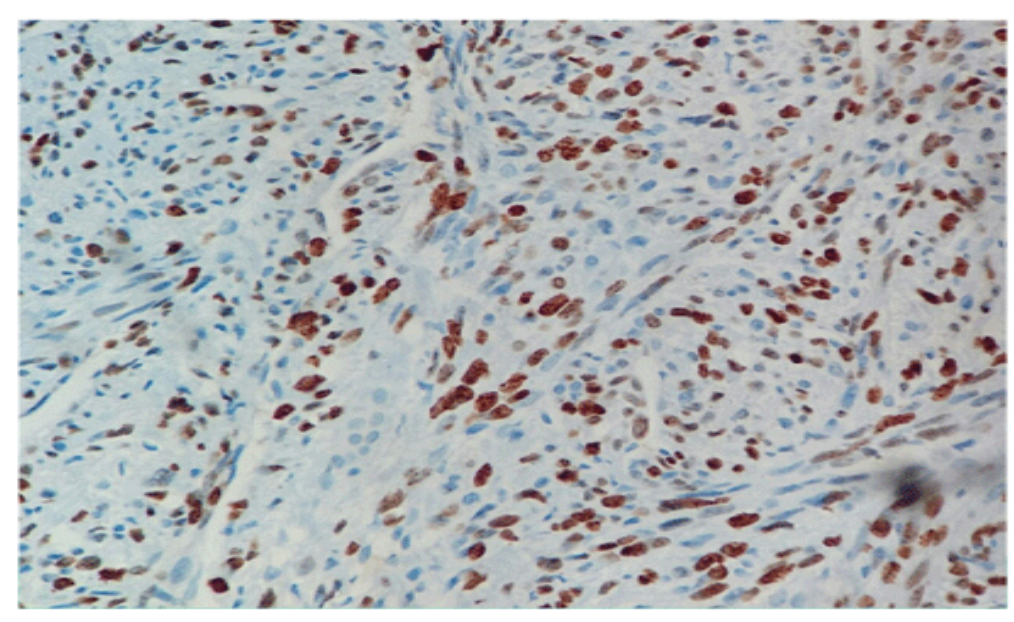

Figure 5: Immunohistochemistry: CD31: negative, H-Caldesmon-patchy positive, Ki67 proliferation index: 40\%, Alk-1: negative

\section{Discussion}

Primary hepatic leiomyosarcoma is a rare cause of primary hepatic malignancies, accounting for less than $2 \%$ of all primary liver malignancies [1]. The commonest cause of hepatic leiomyosarcomas is metastasis from a primary in the lungs, gastrointestinal tract, uterus and peritoneum [2]. Hence exclusion of metastasis is important prior to making a diagnosis of a primary.

Primary leiomyosarcoma of the liver arises from smooth muscle cells in the wall of intrahepatic blood vessels or biliary ducts $[3,4]$. Commonest primary liver sarcomas are angiosarcoma (36 \%) followed by leiomyosarcoma (12\%), fibrosarcoma (7\%), and other sarcomas (44\%) [5]. Mean age of diagnosis is about 58 years [6]. There is no gender predisposition. Etiology of Primary hepatic leiomyosarcoma has not been conclusively discerned yet, but is said to be more common in immunocompromised individuals such as HIV, hepatitis C with cirrhosis.

The case usually presents with non specific symptoms such as generalized weakness, loss of appetite and involuntary weight loss, along with more specific features such as abdominal pain and swelling. In our case, the patient was symptomatic but the laboratory investigations were inconclusive. The absence of a tumor marker or serology for primary hepatic leiomyosarcoma further makes early diagnosis difficult.

A large, well-defined, heterogeneous hypodense mass with internal and peripheral enhancement or cystic mass with an enhancing thick wall may be seen on CT scan $[7,8,9]$. Immunohistochemistry will stain positive for desmin, vimentin and smooth muscle actin (SMA) will be present but will not stain for cytokeratin, S-100 protein, and neuron-specific enolase [9]. In our case SMA and vimentin were positive but desmin was negative. Also H-Caldesmon was patchy positive which tilted the diagnosis in favour of primary hepatic leiomyosarcoma. Due to the rarity of cases of Primary hepatic leiomyosarcoma, the standard of care has not been ascertained. A large global database is required to formulate management guidelines for primary hepatic leiomyosarcoma. A variety of treatment options have been attempted such as surgery, chemotherapy, surgery with neo-adjuvant chemotherapy and liver transplant. However, no single treatment modality has shown to be effective over the other in treatment of Primary hepatic leiomyosarcoma. Husted et al. conducted a study in which 6 patients with primary hepatic angiosarcoma and 13 patients with metastatic sarcoma underwent liver transplantation and the 1-, 3-, and 5-year survival was $47 \%, 15 \%$ and $5 \%$, respectively. Recurrence rate was $95 \%$ after a median interval of 6 months. In view of the high rate of tumour recurrence, Husted et al discouraged liver transplant as a modality for treating primary hepatic leiomyosarcomas [10].

Irinotecan, a topoisomerase II inhibitor, has shown to be effective in inhibiting the growth of Primary hepatic leiomyosarcomas [11]. Tumour free margins make surgical resection possible. However, the recurrence rates are very high. Our patient was treated with BCCA protocol for sarcomas with intact liver functions, with Adriamycin and Ifosfomide and is on follow up.

\section{Conclusion}

Primary Hepatic Leiomyosarcoma is a rare primary hepatic malignancy which is usually a diagnosis of exclusion. Primary liver malignancies which remain a diagnostic dilemma after laboratory investigations and imaging studies should be subjected to histopathological evaluation after liver biopsy of the lesion and further immunohistochemistry tests. A broader database is required to form guidelines for management of Primary Hepatic Leiomyosarcoma.

\section{References}

1. Maki HS, HubertBC,Sajjad SM, KirchnerJP,Kuehner ME. Primary hepaticleiomyosarcoma. Arch Surg. 1987;122(10):1193-1196. Doi:10.1001/archsurg.1987.01400220103020

2. Cioffi U, Quattrone P, De Simone M, Bonavina L, Segalin A, Masini T, et al. Primary multiple epithelioid leiomyosarcoma of the liver. Hepatogastroenterology. 1996;43(12):16031605.

3. G Civardi, L Cavanna, E Iovine, E Buscarini, D Valus A, L Buscarin. Diagnostic imaging of primary hepatic leiomyosarcoma: a case report. Ital J Gastroenterol. 1996;28(2):98101.

4. H Holloway, CB Walsh, R Thomas, J Fielding. Primary hepatic leiomyosarcoma. Journal of Clinical Gastroenterology. 1996;23(2):131-133.

5. Weitz J, Klimstra DS, Cymes K, Jarnagin WR, D’Angelica M, La Quaglia MP, et al. Management of primary liver sarcomas. Cancer. 2007;109(7):1391-1396. 
6. M Chi, AZ Dudek, KP Wind. Primary hepatic leiomyosarcoma in adults: analysis of prognostic factors. Onkologie. 2012;35:210-214.

7. F Ferrozzi, D Bova, A Zangrandi, G Garlaschi. Primary liver leiomyosarcoma: CT appearance. Abdominal Imaging. 1996;21(2):157-160.

8. P Soyer, DA Bluemke, M Riopel, RH Hruban, EK Fishman. Hepatic leiomyosarcomas: CT features with pathologic correlation. Eur J Radiol. 1995;19(3):177-182.

9. Fujita H, Kiriyama M, Kawamura T, Ii T, Takegawa S, Dohba S, et al. Primary hepatic leiomyosarcoma in a woman after renal transplantation: report of a case. Surg Today. 2002;32(5):446-449.
10.Husted TL, Neff G, Thomas MJ, Gross TG, Woodle ES, Buell JF. Liver transplantation for primary or metastatic sarcoma to the liver. American Journal of Transplantation. Am J Transplant. 2006;6(2):392-397.

11.Takehara K, Aoki H, Takehara Y, Yamasaki R, Tanakaya K, Hitoshi Takeuchi. Primary hepatic leiomyosarcoma with liver metastasis of rectal cancer. World J Gastroenterol. 2012;18(38):5479-5484. Doi: 10.3748/wjg.v18.i38.5479 